\title{
Particle Simulations of Sheath Dynamics in Low Pressure Capacitively Coupled Argon Plasma Discharges
}

\author{
Yoshinori Takao*, Kenji Matsuoka, Koji Eriguchi, and Kouichi Ono \\ Department of Aeronautics and Astronautics, Graduate School of Engineering, \\ Kyoto University, Yoshida-Honmachi, Sakyo-ku, Kyoto 606-8501, Japan
}

A numerical study of low-pressure capacitively coupled plasma (CCP) discharges in Ar has been performed including the transport of ions in a sheath onto a substrate. We employ a two-dimensional axisymmetric particle-in-cell with a Monte Carlo collision (PIC-MCC) method for an asymmetric capacitive discharge with an external electrical circuit containing a blocking capacitor and an rf power supply. The PIC-MCC results show that for the gas pressure $(5-100 \mathrm{mTorr})$, rf voltage $(50-500 \mathrm{~V})$, and frequency $(6.78-40.68 \mathrm{MHz})$ ranges examined for a single-frequency $\mathrm{CCP}$, the peak plasma density increases linearly with gas pressure, rf voltage, and frequency squared. With a decrease in pressure or increases in plasma density and sheath potential, the sheath becomes less collisional and thus significant peaks at high energies of ion energy distributions appear and narrow ion angular distributions are obtained. The tendency is in a reasonable agreement with experimental results.

\footnotetext{
${ }^{*}$ E-mail address: takao.yoshinori.7a@kyoto-u.ac.jp
} 


\section{Introduction}

Controlling feature profiles during plasma etching has been a critical problem of interest in the fabrication of modern microelectronic devices. The etched profiles are strongly dependent on the transport of ions and neutrals onto substrate surfaces through a sheath. The shrinking of device features requires low-pressure plasma etching to reduce the broadening of the ion angular distribution caused by ion-neutral collisions in the sheath. Although capacitively coupled plasma (CCP) discharges are widely used for plasma etching, CCP discharges at low pressures $(<30$ mTorr $)$ are not studied intensively compared with those at middle and high pressures. ${ }^{1)}$ Since kinetic effects play a dominant role at low pressures, one should employ a particle model rather than a fluid model to investigate the discharge and

sheath dynamics numerically. A number of one-dimensional particle simulations were conducted for CCP discharges. ${ }^{1-7)}$ However, only few studies have been carried out using a particle model with more than one dimension. ${ }^{8)}$

In this paper, a numerical study on low-pressure asymmetric (i.e., a large difference in size between rf-powered and grounded electrodes) CCP discharges in Ar has been conducted to analyze the transport of charged particles in the sheath. We employ a two-dimensional axisymmetric particle-in-cell with a Monte Carlo collision (PIC-MCC) method, ${ }^{9-11)}$ with an external electrical circuit containing a blocking capacitor and an rf power supply. Thus, the model gives self-consistently the dc self-bias voltages generated on the rf-powered electrode and the energy and angular distributions of charged particles incident onto the substrate surfaces.

The numerical model is briefly described in $\S 2$, and then results and discussion are presented in $\S 3$, where attention is focused on the effects of the gas pressure, rf voltage, and 
frequency on the structure of plasma discharges, and the energy and angular distribution functions of ions (IEDF and IADF) incident onto the substrate. Section 4 shows the conclusions of this study.

\section{Numerical Model}

Figure 1 shows a schematic of the parallel-plate CCP reactor of interest in this study. The reactor consists of a grounded cylindrical chamber $25 \mathrm{~cm}$ in diameter and $4 \mathrm{~cm}$ in height, having an rf-powered electrode $10 \mathrm{~cm}$ in diameter at the bottom of the chamber. Thus, there is a large difference in size between the rf-powered electrode and grounded wall, i.e., the discharge system is asymmetric. ${ }^{12)}$ The powered electrode is coupled to an rf power supply through a blocking capacitor of capacitance $C_{\mathrm{B}}=500 \mathrm{pF}$, and the working gas is Ar. The time-varying voltage of the rf source is taken as $V_{\mathrm{s}}(t)=V_{\mathrm{rf}} \cos \left(2 \pi f_{\mathrm{rf}} t\right)$ with the rf voltage $V_{\mathrm{rf}}$ and frequency $f_{\mathrm{rf}}$.

The plasma simulation employs a two-dimensional PIC-MCC method, in which a number of simulated particles (i.e., superparticles for electrons and ions) are loaded into a two-dimensional spatial computational mesh $(r, z)$, along with three velocity components $\left(v_{r}\right.$, $v_{\theta}$, and $v_{z}$ ). Our PIC-MCC model for charged species (singly ionized $\mathrm{Ar}^{+}$and electrons) generally follows the methodology described in ref. 9 and references therein; thus, only a brief description of the computational technique for CCP discharges is included here.

The equation of motion for particles is given by

$$
m \frac{d \boldsymbol{v}}{d t}=-q \frac{\partial \phi}{\partial \boldsymbol{r}}
$$

where $m$ is the particle mass, $\boldsymbol{v}=d \boldsymbol{r} / d t$ the velocity, $q$ the charge, $\boldsymbol{r}$ the position, and $\phi$ the local electrostatic potential. The numerical time step $\Delta t$ is taken to be $1.475 \times 10^{-11} \mathrm{~s}(1 / 5000$ 
of an rf cycle for $13.56 \mathrm{MHz})$ for electrons and $3.687 \times 10^{-10} \mathrm{~s}(1 / 200$ of an $\mathrm{rf}$ cycle) for ions, according to their difference in the speed of motion.

The reactions taken into account are elastic scattering, excitation, and ionization for electrons, and elastic scattering and charge exchange for ions, ${ }^{13-16)}$ which are described by the null-collision method; ${ }^{16)}$ moreover, secondary electron emissions are also included with an emission coefficient $\gamma=0.1$, through energetic ion bombardment on powered electrode surfaces. The data on the cross sections for electron-neutral and ion-neutral collisions are the same as those used in ref. 9. In addition, neutral particles are assumed to be uniform in the discharge region throughout the simulation with a Maxwellian velocity distribution at a gas temperature of $300 \mathrm{~K}(=0.026 \mathrm{eV})$. The motion of excited-state atoms and Coulomb collisions between charged particles are not considered in this study.

The potential $\phi$ in the discharge is derived from the Poisson equation in the cylindrical coordinates. The computational region is divided into equally spaced 400 grids along the $r$-axis and 128 grids along the $z$-axis. For the plasma parameter range calculated in the present study, the grid spacing $(\Delta r, \Delta z)$ is sufficiently small to satisfy the following condition: the Debye length $\lambda_{\mathrm{D}}$ is larger than $\Delta r / 3, \Delta z / 3 .{ }^{17)}$ The equation is solved with boundary conditions $\phi=0$ at the chamber walls and $\phi=V$ at the rf-powered electrode, where $V$ is determined by calculating the voltage and current in the external electrical circuit at every time step for ions. ${ }^{18,19)}$

The current $I$ in the circuit is calculated by the conservation of charge at the rf-powered electrode:

$$
I=I_{\mathrm{d}}+I_{\mathrm{c}}=\int_{S} \varepsilon_{0} \frac{\partial E_{z}}{\partial t} d A+I_{\mathrm{c}}
$$

where $I_{d}$ is the displacement current, and $I_{c}$ is the conduction current, which consists of the 
flux of ions $I_{i}$ and electrons $I_{e}$ from the plasma onto the electrode surfaces and the flux of secondary electrons therefrom. Note that $\int_{S} d A$ denotes the surface integral over the powered electrode surfaces, and the electric field thereat is given by $E_{z}=-d \phi / d z$. The electrode potential or voltage $V$ at the new time step is calculated based on the circuit equation

$$
V=V_{\mathrm{S}}(t)-\frac{Q}{C_{\mathrm{B}}}=V_{\mathrm{S}}(t)-\frac{1}{C_{\mathrm{B}}} \int_{T} I d t
$$

where $Q$ denotes the charge stored in the blocking capacitor. Then, the Poisson equation is solved with the $V$ given by eq. (3) as the boundary condition at the new time step. Thus, we take into account the effects of the external circuit on the potential distribution in the discharge, which in turn gives the dc self-bias voltages self-consistently after the iteration of these calculations until the system reaches a steady state.

\section{Results and Discussion}

An argon discharge was calculated for the gas pressure $p=20$ mTorr, rf voltage $V_{\text {rf }}=100 \mathrm{~V}$, and $\mathrm{rf}$ frequency $f_{\mathrm{rf}}=13.56 \mathrm{MHz}$. Figure 2 shows the two-dimensional distribution of the electron density $n_{\mathrm{e}}$ and potential $\phi$ in the plasma reactor, exhibiting significant sheath structures around the rf-powered electrode. The peak electron density obtained in the discharge is $2.9 \times 10^{8} \mathrm{~cm}^{-3}$, where the plasma potential $\phi_{\mathrm{p}}$ and dc self-bias voltage $V_{\mathrm{dc}}$ are 28 and $-66 \mathrm{~V}$, respectively. Figure 3 shows the time-varying behavior of the currents of the displacement $I_{\mathrm{d}}$, ion $I_{\mathrm{i}}$, and electron $I_{\mathrm{e}}$ at the powered electrode, together with the potentials $V$ thereat and $\phi_{\mathrm{p}}$ in the discharge (at $r=0, z=2 \mathrm{~cm}$ ), corresponding to the situation in Fig. 2 . The incoming flux $I_{\mathrm{e}}$ of plasma electrons onto the powered electrode occurs only around the period when the electrode potential $V$ reaches its peak and the difference between the plasma and electrode potentials $\phi_{\mathrm{p}}-V$ is minimum; in contrast, the influx $I_{\mathrm{i}}$ of ions remains almost 
constant throughout the rf cycle. The total circuit current $I$ is almost equal to the displacement current $I_{\mathrm{d}}$ except when the electron current $I_{\mathrm{e}}$ is observed.

\subsection{Pressure dependence}

The electron density increases almost linearly with an increase in gas pressure. The peak electron densities obtained at $V_{\mathrm{rf}}=100 \mathrm{~V}$ and $f_{\mathrm{rf}}=13.56 \mathrm{MHz}$ are $9.3 \times 10^{7}, 1.6 \times 10^{8}, 8.2 \times 10^{8}$, and $2.1 \times 10^{9} \mathrm{~cm}^{-3}$ for $p=5,10,50$, and 100 mTorr, respectively. Figure 4 shows the two-dimensional distribution of $n_{\mathrm{e}}$ and $\phi$ in the plasma reactor at $V_{\mathrm{rf}}=100 \mathrm{~V}$ and $f_{\mathrm{rf}}=13.56$ MHz for $p=5$ mTorr, and that for $p=100$ mTorr is shown in Fig. 5. As shown in Figs. 2, 4, and 5, increasing gas pressure results in a narrow sheath structure owing to the increase in electron density. With increasing pressure from 5 to $100 \mathrm{mTorr}$, the plasma potential $\phi_{\mathrm{p}}$ decreases from 38 to $26 \mathrm{~V}$, while the dc self-bias voltage $V_{\mathrm{dc}}$ values are -62 and $-64 \mathrm{~V}$ for 5 and 100 mTorr, respectively, which are almost constant.

Figure 6 shows the normalized IEDFs and IADFs incident onto the rf-powered electrode for $p=5,10,20,50$, and 100 mTorr, calculated at $V_{\mathrm{rf}}=100 \mathrm{~V}$ and $f_{\mathrm{rf}}=13.56 \mathrm{MHz}$. Here, the normalized distribution function $f(x)$ is defined as $\int f(x) d x=1 \quad(x=\varepsilon$, ion energy in electron volt for IEDF, and $x=\theta$, angle in degree for IADF). For $p=5$ mTorr, the sheath is almost collisionless and most ions reach the powered electrode with the time-averaged sheath potential; thus, the IEDF has a significant peak at an ion energy of about $100 \mathrm{eV}$. With an increase in pressure, the high energy peak vanishes, a large fraction of low energy ions is obtained, and then the low energy peak appears. This implies that at higher pressures, ion-neutral collisions occur more frequently during the ion acceleration through the sheath onto the electrode, leading to the decreased ion energies. As shown in Fig. 6(b), most ions 
impact the electrode almost perpendicularly after being accelerated through the sheath (or at the incident angle $\theta \sim 0^{\circ}$ from the electrode surface normal). The peak value of IADFs decreases with increasing pressure above 20 mTorr, implying that at higher pressures, the incident ions are subject to the acceleration through the narrower, lower-voltage sheath with more frequent ion-neutral collisions. However, below 20 mTorr, larger distributions are obtained with a decrease in pressure at $\theta=2-5^{\circ}$. This result would be due to the thick sheath at low pressures, leading to the high curvature of equipotential lines around the edge of the electrode, as shown in Fig. 4(b). It should be noted that the definition of IADFs is the same as that used by Lee et al. ${ }^{6)}$ Since the circular differential area on the unit sphere at an incident angle $\theta$ is defined as $d A=2 \pi \sin \theta d \theta, d A$ decreases with decreasing $\theta$, so that IADFs also approach zero at $\theta \sim 0^{\circ}$. The pressure dependence of IEDFs and IADFs is qualitatively consistent with experimental results. ${ }^{20-23)}$

\subsection{Voltage dependence}

Increasing rf voltage results in a high electron density. The peak electron densities obtained at $p=20 \mathrm{mTorr}$ and $f_{\mathrm{rf}}=13.56 \mathrm{MHz}$ are $1.4 \times 10^{8}, 9.1 \times 10^{8}$, and $1.6 \times 10^{9} \mathrm{~cm}^{-3}$ for $V_{\mathrm{rf}}=50,300$, and $500 \mathrm{~V}$, respectively. The electron density increases almost linearly with rf voltage. Figure 7 shows the two-dimensional distribution of $n_{\mathrm{e}}$ and $\phi$ in the plasma reactor for $p=20$ mTorr, $V_{\mathrm{rf}}=500 \mathrm{~V}$, and $f_{\mathrm{rf}}=13.56 \mathrm{MHz}$, where $\phi_{\mathrm{p}}=39 \mathrm{~V}$ and $V_{\mathrm{dc}}=-418 \mathrm{~V}$. Compared with Fig. 2, a significantly wide sheath structure can be seen owing to the large potential difference between $\phi_{\mathrm{p}}$ and $V_{\mathrm{dc}}$.

Figure 8 shows the normalized IEDFs and IADFs incident onto the rf-powered electrode for different $V_{\text {rf }}$ values in the range from 50 to $500 \mathrm{~V}$, calculated at $p=20$ mTorr 
and $f_{\mathrm{rf}}=13.56 \mathrm{MHz}$. As shown in Fig. 8(a), the peak at the highest energy of each IEDF is obtained at the ion energy of approximately $\phi_{\mathrm{p}}+\left|V_{\mathrm{dc}}\right|$. Since the mean free path of ions $\lambda_{\mathrm{i}}$ is about $0.15 \mathrm{~cm}$ at $p=20$ mTorr $\left(\lambda_{\mathrm{i}}=1 / 330 p \mathrm{~cm}, p\right.$ in Torr $\left.{ }^{12)}\right)$ and the sheath width is determined to be more than $0.5 \mathrm{~cm}$ as shown in Figs. 2 and 7, most ions collide with neutrals through the sheath. In our PIC-MCC model, charge exchange collisions are included as stated in $\S 2$. Once an ion accelerated in the sheath undergoes a charge exchange collision, the ion picks up an electron from a slow gas atom and leaves behind a slow scattered ion. ${ }^{11)}$ The slow ion is scattered isotropically with the temperature of $300 \mathrm{~K}(=0.026 \mathrm{eV}) .{ }^{16)}$ Since ions with lower speed have larger cross sections for the charge exchange, lower rf voltages result in many collisions in the sheath and then produce a large fraction of low energy ions, which can be clearly seen in Fig. 8(a). For $V_{\text {rf }}=300$ and $500 \mathrm{~V}$, one can recognize several peaks in the range of ion energies from 0 to $500 \mathrm{~V}$, which are caused by charge exchange collisions for ions in the sheath. ${ }^{22)}$ The IADFs have a significant peak at around normal incidence. With an increase in rf voltage, the peak value of IADFs increases at lower incident angles due to the large sheath potential and few collisions in the sheath compared with lower rf voltages.

\subsection{Frequency dependence}

A higher rf frequency leads to a larger electron density. The peak electron densities obtained at $p=20$ mTorr and $V_{\mathrm{rf}}=100 \mathrm{~V}$ are $1.1 \times 10^{8}, 9.8 \times 10^{8}$, and $2.1 \times 10^{9} \mathrm{~cm}^{-3}$ for $f_{\mathrm{rf}}=6.78,27.12$, and 40.68 MHz, respectively. The electron density increases almost linearly with rf frequency squared, which is in good agreement with the frequency scaling. ${ }^{12)}$ Figure 9 shows the two-dimensional distribution of $n_{\mathrm{e}}$ and $\phi$ in the plasma reactor for $p=20 \mathrm{mTorr}, V_{\mathrm{rf}}$ 
$=100 \mathrm{~V}$, and $f_{\mathrm{rf}}=40.68 \mathrm{MHz}$, where $\phi_{\mathrm{p}}=28 \mathrm{~V}$ and $V_{\mathrm{dc}}=-70 \mathrm{~V}$. The narrow sheath and large negative dc self-bias voltage can be seen owing to the increase in electron density by one order of magnitude compared with that shown in Fig. 2.

Figure 10 shows the normalized IEDFs and IADFs incident onto the rf-powered electrode for $f_{\mathrm{rf}}=6.78,13.56,27.12$, and $40.68 \mathrm{MHz}$, calculated at $p=20$ mTorr and $V_{\mathrm{rf}}=$ $100 \mathrm{~V}$. The IEDFs and IADFs change markedly as the rf frequency increases. With increasing rf frequency, the high energy peak appears clearly for IEDFs and the peak value of IADFs increases at lower incident angles. At high frequencies, the sheath width decreases and is comparable to the ion mean free path; thus, many ions fall through the sheath to the powered electrode without collisions in contrast to the case of $f_{\mathrm{rf}}=13.56 \mathrm{MHz}$ or less. This implies that the IEDF and IADF can also be controlled by the driven frequency.

\subsection{Dual frequency CCP: low-frequency voltage dependence}

Finally, we have investigated the low-frequency voltage dependence for a dual-frequency CCP. A low-frequency rf voltage $V_{\mathrm{LF}}$ is superimposed on a high-frequency rf voltage $V_{\mathrm{HF}}$ : $V_{\mathrm{s}}(t)=V_{\mathrm{HF}} \cos \left(2 \pi f_{\mathrm{HF}} t\right)+V_{\mathrm{LF}} \cos \left(2 \pi f_{\mathrm{LF}} t\right)$, where $f_{\mathrm{HF}}=27.12 \mathrm{MHz}$ and $f_{\mathrm{LF}}=f_{\mathrm{HF}} / 14=1.937$

MHz. Figure 11(a) shows the peak ion density $n_{\text {peak }}$ and ion flux $\Gamma_{\text {ion }}$ incident onto the electrode as a function of low-frequency voltage $V_{\mathrm{LF}}$ for $V_{\mathrm{HF}}=100 \mathrm{~V}$ and $p=20$ mTorr. Although the peak density $n_{\text {peak }}$ decreases monotonically with increasing $V_{\mathrm{LF}},{ }^{6}$ the ion flux $\Gamma_{\text {ion }}$ decreases below $V_{\mathrm{LF}}=100 \mathrm{~V}$ and then levels off. Increasing low-frequency voltage leads to a high ion velocity incident onto the powered electrode, which compensates the decrease in electron density and then produces constant ion fluxes.

Figures 11(b) and 11(c) show the normalized IEDFs and IADFs incident onto the 
rf-powered electrode for different $V_{\mathrm{LF}}$ values in the range from 0 to $300 \mathrm{~V}$. Increasing low-frequency voltage results in the disappearance of the high energy peak of IEDFs. This tendency is consistent with one-dimensional PIC-MCC calculations. ${ }^{5,6,8)}$ In contrast to the voltage dependence of the single-frequency $\mathrm{CCP}$ as shown in Fig. 8, the electron density decreases with increasing $V_{\mathrm{LF}}$. This indicates that the sheath thickness increases and is much larger than the ion mean free path, which results in a more collisional sheath. In addition, the ion plasma frequency for Ar is $1.1 \mathrm{MHz}$ at the plasma density of $10^{9} \mathrm{~cm}^{-3}$, which is less than the low frequency $f_{\mathrm{LF}}=1.937 \mathrm{MHz}$. Since the plasma density decreases with increasing $V_{\mathrm{LF}}$, i.e., the ion plasma frequency decreases, ions do not completely follow the instantaneous sheath potential; thus, there is a small distribution at higher ion energies. The peak value of IADFs increases at lower incident angles with increasing $V_{\mathrm{LF}}$, even though the sheath becomes more collisional. This implies that the sheath potential dominantly contributes to IADFs. In the dual-frequency CCP examined in this study, there is a small process window where an ion flux is independent of low-frequency voltage, as indicated in one-dimensional PIC-MCC simulations. ${ }^{24)}$

\section{Conclusions}

A two-dimensional axisymmetric PIC-MCC method for an asymmetric capacitive discharge with an external electric circuit has been employed to investigate the transport of ions in the sheath of a low-pressure CCP in Ar, where the electron density is less than $10^{10} \mathrm{~cm}^{-3}$. The model gives self-consistently the dc self-bias voltages typically generated on the rf-powered electrode, along with the time-varying behavior of the voltage and current thereat, and the energy and angular distributions of ions (IEDF and IADF) incident onto substrate surfaces for 
various process conditions of a single-frequency CCP. The peak electron density obtained in the discharge is $2.9 \times 10^{8} \mathrm{~cm}^{-3}$ at the Ar gas pressure of $20 \mathrm{mTorr}$, rf frequency of $13.56 \mathrm{MHz}$, and rf voltage of $100 \mathrm{~V}$, where the dc self-bias voltage is determined to be $-66 \mathrm{~V}$. For the gas pressure (5-100 mTorr), rf voltage (50-500 V), and frequency $(6.78-40.68 \mathrm{MHz})$ ranges examined, the peak electron density increases linearly with pressure, $r f$ voltage, and frequency squared. The IEDFs and IADFs are strongly dependent on the plasma density, in addition to the sheath potential. With a decrease in pressure or increases in plasma density and sheath potential, the sheath becomes less collisional and thus significant peaks at high energies of IEDFs appear and narrow IADFs are obtained. The tendency is qualitatively consistent with experimental results. For a dual-frequency CCP, a small process window where an ion flux is independent of low-frequency voltage is determined. Since the electron density calculated in the present work is much lower than that used in actual plasma processing, the results and discussion of this study is limited to low-density plasmas and thus particle simulations of high-density plasmas are left for future work. Nonetheless, the information on IEDFs and IADFs obtained in the study will be useful for etching profile simulations ${ }^{25,26)}$ and the analysis of plasma-induced damage. ${ }^{27,28)}$

\section{Acknowledgments}

We thank Dr. Y. Doi, Mr. T. Fujiwara, Mr. T. Nomura, Dr. Y. Osano, and Mr. T. Suzumura, who used to be members of our laboratory, for the refinement of the simulation code. This work was financially supported in part by a Grant-in-Aid for Scientific Research on Innovative Areas (21110008) from the Ministry of Education, Culture, Sports, Science and Technology. 
1) S. Rauf, K. Bera, and K. Collins: Plasma Sources Sci. Technol. 19 (2010) 015014.

2) J. Schulze, Z. Donkó, D. Luggenhölscher, and U. Czarnetzki: Plasma Sources Sci. Technol. 18 (2009) 034011.

3) S. H. Lee, P. K. Tiwari, and J. K. Lee: Plasma Sources Sci. Technol. 18 (2009) 025024.

4) A. C. F. Wu, M. A. Lieberman, and J. P. Verboncoeur: J. Appl. Phys. 101 (2007) 056105 .

5) Y. J. Hong, H. S. Ko, G. Y. Park, and J. K. Lee: Comput. Phys. Commun. 177 (2007) 122.

6) J. K. Lee, O. V. Manuilenko, N. Y. Babaeva, H. C. Kim, and J. W. Shon: Plasma Sources Sci. Technol. 14 (2005) 89.

7) V. Georgieva and A. Bogaerts: J. Appl. Phys. 98 (2005) 023308.

8) G. Wakayama and K. Nanbu: IEEE Trans. Plasma Sci. 31 (2003) 638.

9) Y. Takao, N. Kusaba, K. Eriguchi, and K. Ono: J. Appl. Phys. 108 (2010) 093309.

10) K. Nanbu: IEEE Trans. Plasma Sci. 28 (2000) 971.

11) C. K. Birdsall: IEEE Trans. Plasma Sci. 19 (1991) 65.

12) M. A. Lieberman and A. J. Litchenberg: Principles of Plasma Discharges and Materials Processing (Wiley, New York, 1994).

13) A. Bogaerts, R. Gijbels, and W. Goedheer: Jpn. J. Appl. Phys. 38 (1999) 4404.

14) W. H. Cramer: J. Chem. Phys. 30 (1959) 641.

15) M. Surendra, D. B. Graves, and G. M. Jellum: Phys. Rev. A 41 (1990) 1112.

16) V. Vahedi and M. Surendra: Comput. Phys. Commun. 87 (1995) 179.

17) C. K. Birdsall and A. B. Langdon: Plasma Physics via Computer Simulation (IoP Publishing, Bristol, U.K., 1991) p. 308. 
18) J. P. Verboncoeur, M. V. Alves, V. Vahedi, and C. K. Birdsall: J. Comput. Phys. 104 (1993) 321.

19) V. Vahedi and G. DiPeso: J. Comput. Phys. 131 (1997) 149.

20) J. Schulze, E. Schüngel, and U. Czarnetzki: J. Phys. D 42 (2009) 092005.

21) U. Flender and K. Wiesemann: J. Phys. D 27 (1994) 509.

22) C. Wild and P. Koidl: J. Appl. Phys. 69 (1991) 2909.

23) J. R. Woodworth, M. E. Riley, D. C. Meister, B. P. Aragon, M. S. Le, and H. H. Sawin: J. Appl. Phys. 80 (1996) 1304.

24) Z. Donkó, J. Schulze, P. Hartmann, I. Korolov, U. Czarnetzki, and E. Schüngel: Appl. Phys. Lett. 97 (2010) 081501.

25) H. Tsuda, M. Mori, Y. Takao, K. Eriguchi, and K. Ono: Thin Solid Films 518 (2010) 3475 .

26) H. Tsuda, M. Mori, Y. Takao, K. Eriguchi, and K. Ono: Jpn. J. Appl. Phys. 49 (2010) 08JE01.

27) K. Eriguchi, Y. Nakakubo, A. Matsuda, Y. Takao, and K. Ono: Jpn. J. Appl. Phys. 49 (2010) 056203.

28) A. Matsuda, Y. Nakakubo, Y. Takao, K. Eriguchi, and K. Ono: Thin Solid Films 518 (2010) 3481. 


\section{List of Figure Captions}

Fig. 1. Schematic of the asymmetric parallel-plate $\mathrm{rf}$ plasma reactor used in the present simulation study, where $\phi_{\mathrm{p}}$ denotes the plasma potential, $V$ and $I$ the voltage and current at the rf-powered electrode, respectively, $C_{\mathrm{B}}$ the capacitance of the blocking capacitor, and $V_{\mathrm{s}}$ the voltage at the rf power source. The PIC-MCC simulation area is $4 \mathrm{~cm}$ in height and $12.5 \mathrm{~cm}$ in radius. The cylindrical coordinates $(r-z)$ are employed, with the origin being placed on the $z$-axis at the bottom of the plasma source (at the top of the rf-powered electrode).

Fig. 2. Spatial distribution of the (a) electron density $n_{\mathrm{e}}$ and (b) potential $\phi$ in the plasma reactor averaged over $100 \mathrm{rf}$ cycles for $p=20 \mathrm{mTorr}, V_{\mathrm{rf}}=100 \mathrm{~V}$, and $f_{\mathrm{rf}}=13.56 \mathrm{MHz}$.

Fig. 3. Time-varying behavior of the displacement $I_{\mathrm{d}}$, ion $I_{\mathrm{i}}$, electron current $I_{\mathrm{e}}$, potential $V$ at the rf-powered electrode, and the plasma potential $\phi_{\mathrm{p}}$ at $r=0, z=2 \mathrm{~cm}$ in one rf cycle under the same conditions as those in Fig. 2.

Fig. 4. Spatial distribution of the (a) electron density $n_{\mathrm{e}}$ and (b) potential $\phi$ in the plasma reactor averaged over $100 \mathrm{rf}$ cycles for $p=5 \mathrm{mTorr}, V_{\mathrm{rf}}=100 \mathrm{~V}$, and $f_{\mathrm{rf}}=13.56 \mathrm{MHz}$.

Fig. 5. Spatial distribution of the (a) electron density $n_{\mathrm{e}}$ and (b) potential $\phi$ in the plasma reactor averaged over $100 \mathrm{rf}$ cycles for $p=100 \mathrm{mTorr}, V_{\mathrm{rf}}=100 \mathrm{~V}$, and $f_{\mathrm{rf}} 13.56 \mathrm{MHz}$.

Fig. 6. Normalized (a) IEDFs and (b) IADFs incident onto the rf-powered electrode $(r=0-5$ 
$\mathrm{cm}$ ) for $p=5,10,20,50$, and $100 \mathrm{mTorr}$, calculated at $V_{\mathrm{rf}}=100 \mathrm{~V}$ and $f_{\mathrm{rf}}=13.56 \mathrm{MHz}$.

Fig. 7. Spatial distribution of the (a) electron density $n_{\mathrm{e}}$ and (b) potential $\phi$ in the plasma reactor averaged over $100 \mathrm{rf}$ cycles for $p=20 \mathrm{mTorr}, V_{\mathrm{rf}}=500 \mathrm{~V}$, and $f_{\mathrm{rf}}=13.56 \mathrm{MHz}$.

Fig. 8. Normalized (a) IEDFs and (b) IADFs incident onto the rf-powered electrode $(r=0-5$ $\mathrm{cm})$ for $V_{\mathrm{rf}}=50,100,300$, and $500 \mathrm{~V}$, calculated at $p=20 \mathrm{mTorr}$ and $f_{\mathrm{rf}}=13.56 \mathrm{MHz}$.

Fig. 9. Spatial distribution of the (a) electron density $n_{\mathrm{e}}$ and (b) potential $\phi$ in the plasma reactor averaged over $100 \mathrm{rf}$ cycles for $p=20 \mathrm{mTorr}, V_{\mathrm{rf}}=100 \mathrm{~V}$, and $f_{\mathrm{rf}}=40.68 \mathrm{MHz}$.

Fig. 10. Normalized (a) IEDFs and (b) IADFs incident onto the rf-powered electrode $(r=$ $0-5 \mathrm{~cm})$ for $f_{\mathrm{rf}}=6.78,13.56,27.12$, and $40.68 \mathrm{MHz}$, calculated at $p=20 \mathrm{mTorr}$ and $V_{\mathrm{rf}}=100$ $\mathrm{V}$.

Fig. 11. (a) Peak ion density $n_{\text {peak }}$ and ion flux $\Gamma_{\text {ion }}$ reaching the rf-powered electrode $(r=$ $0-5 \mathrm{~cm})$ as a function of low-frequency $(1.937 \mathrm{MHz})$ voltage $V_{\mathrm{LF}}$. Normalized (b) IEDFs and (c) IADFs incident onto the rf-powered electrode $(r=0-5 \mathrm{~cm})$ for $V_{\mathrm{LF}}=0,50,100$, and $300 \mathrm{~V}$. Here, the high-frequency $(27.12 \mathrm{MHz})$ voltage $V_{\mathrm{HF}}=100 \mathrm{~V}$ and $p=20 \mathrm{mTorr}$. 


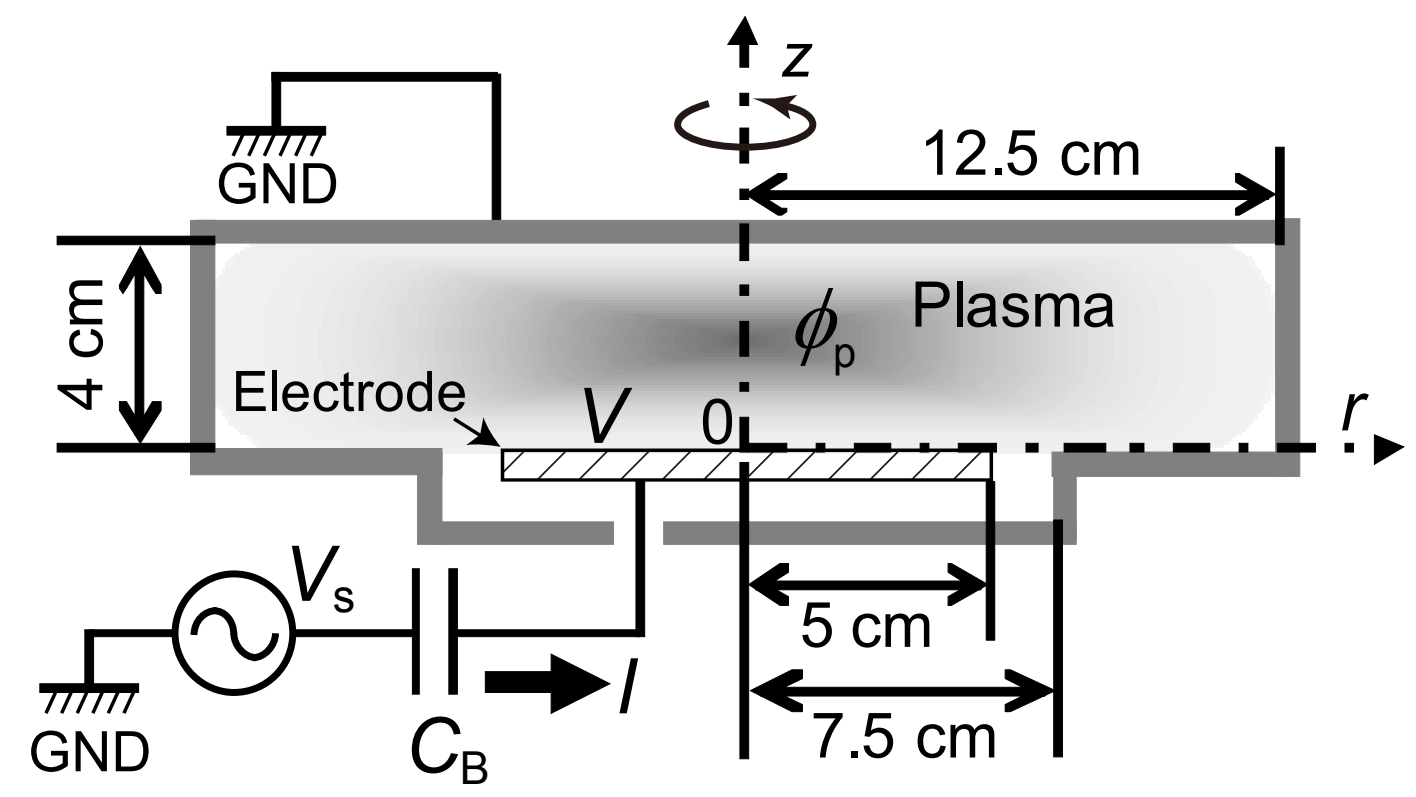

Figure 1 (width $7.0 \mathrm{~cm}$, scale 2/1)

Y. Takao et al. 


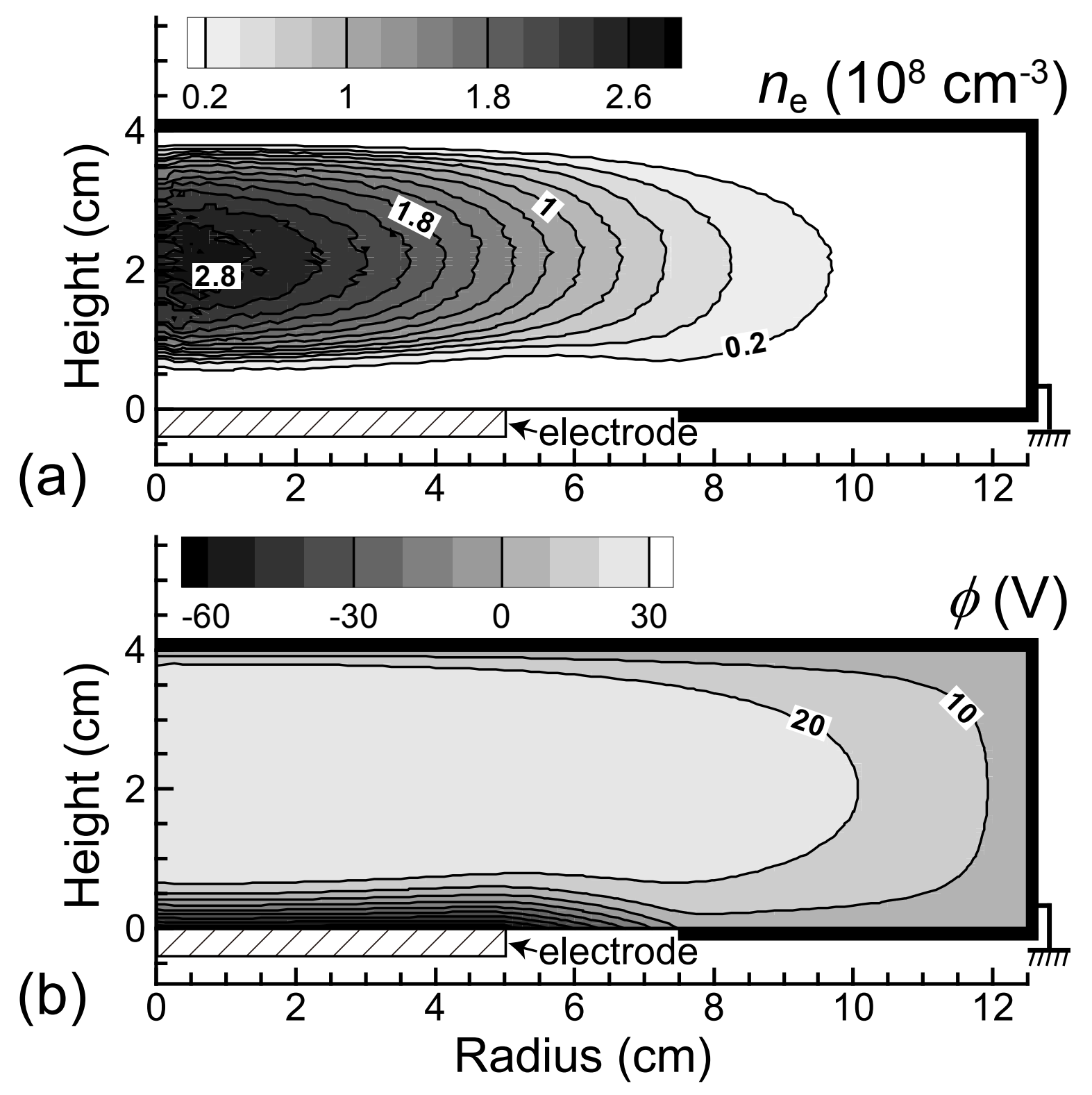

Figure 2 (width $7.7 \mathrm{~cm}$, scale 2/1)

Y. Takao et al. 


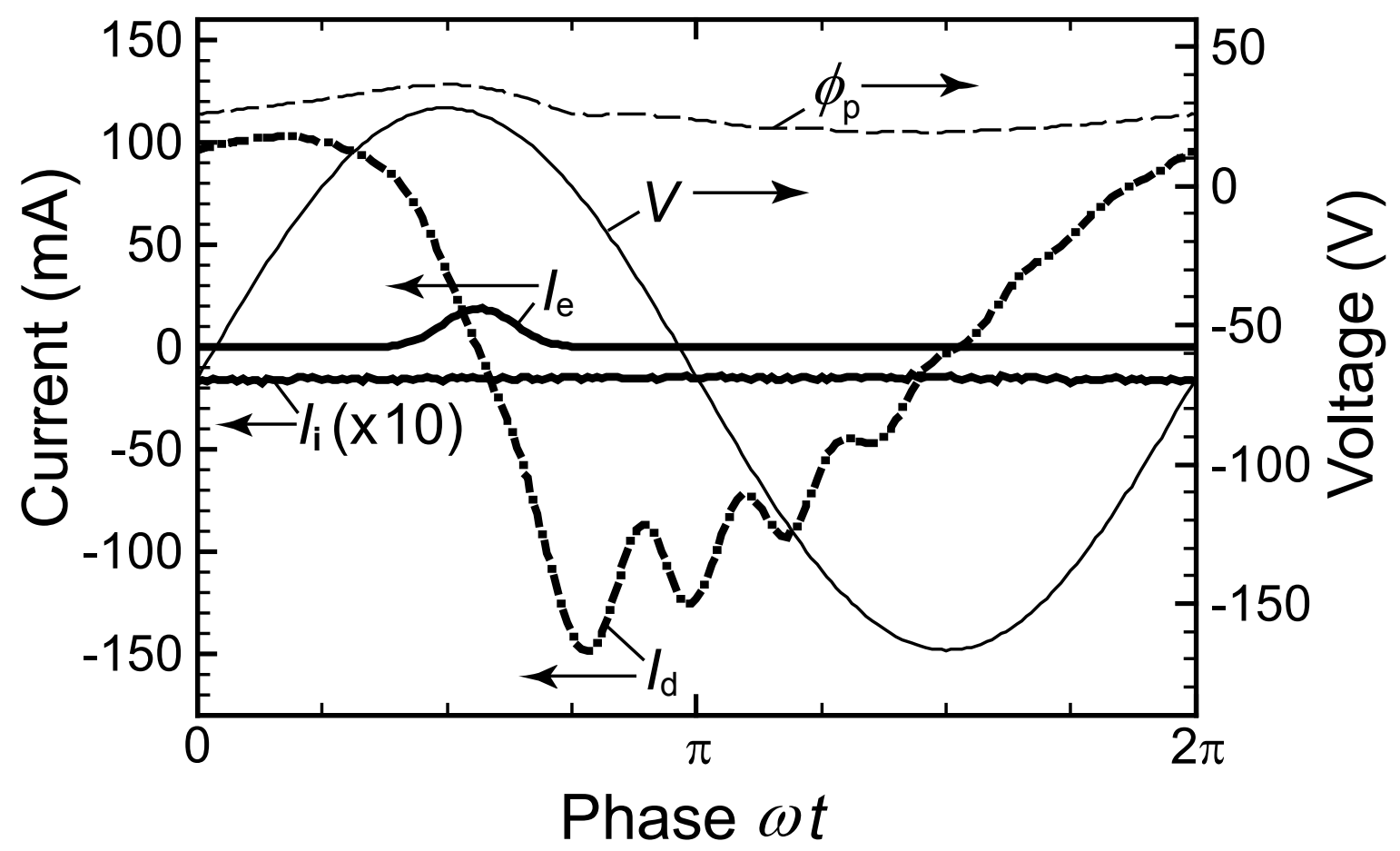

Figure 3 (width $8.0 \mathrm{~cm}$, scale 2/1)

Y. Takao et al. 


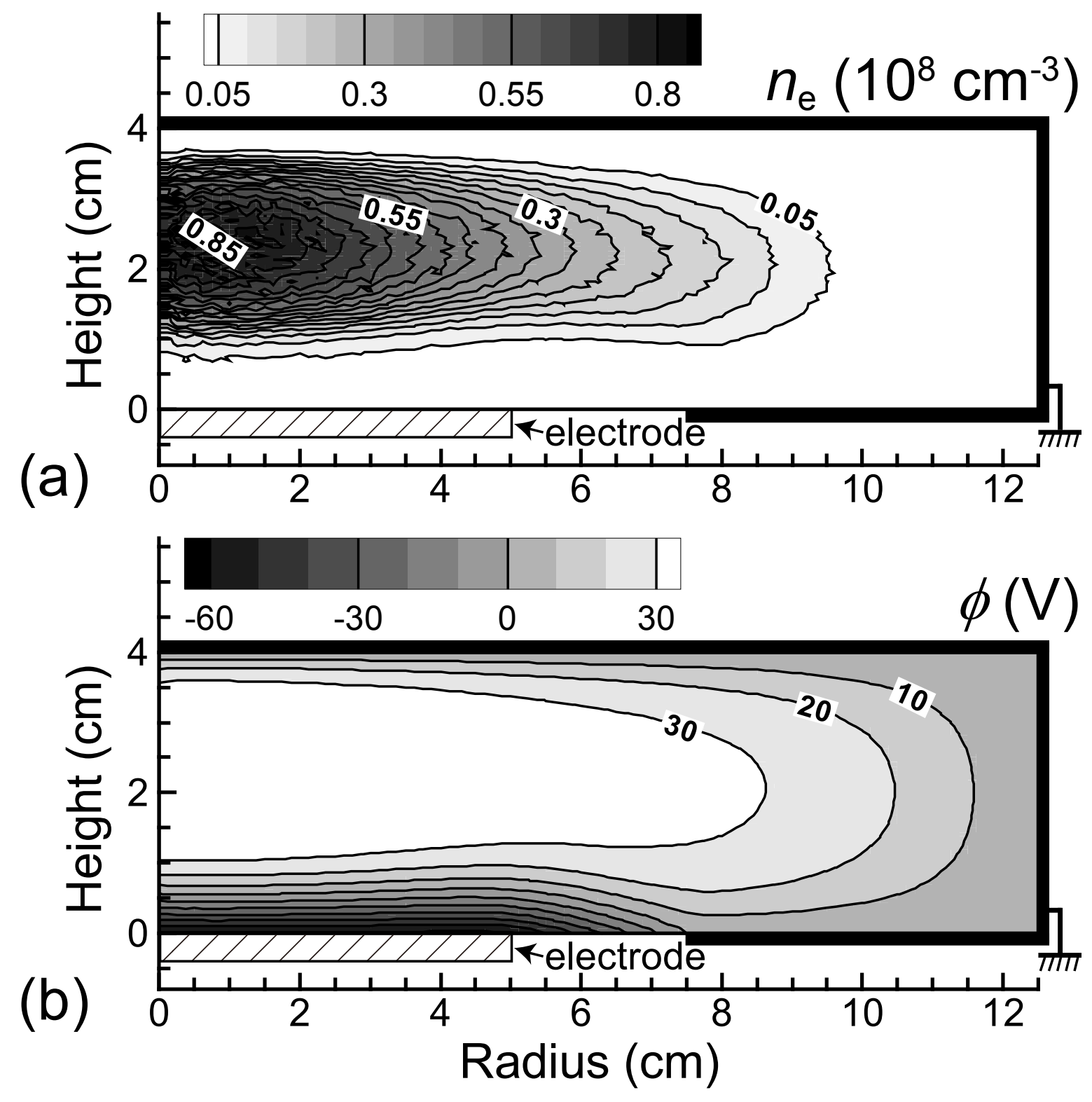

Figure 4 (width $7.7 \mathrm{~cm}$, scale 2/1)

Y. Takao et al. 


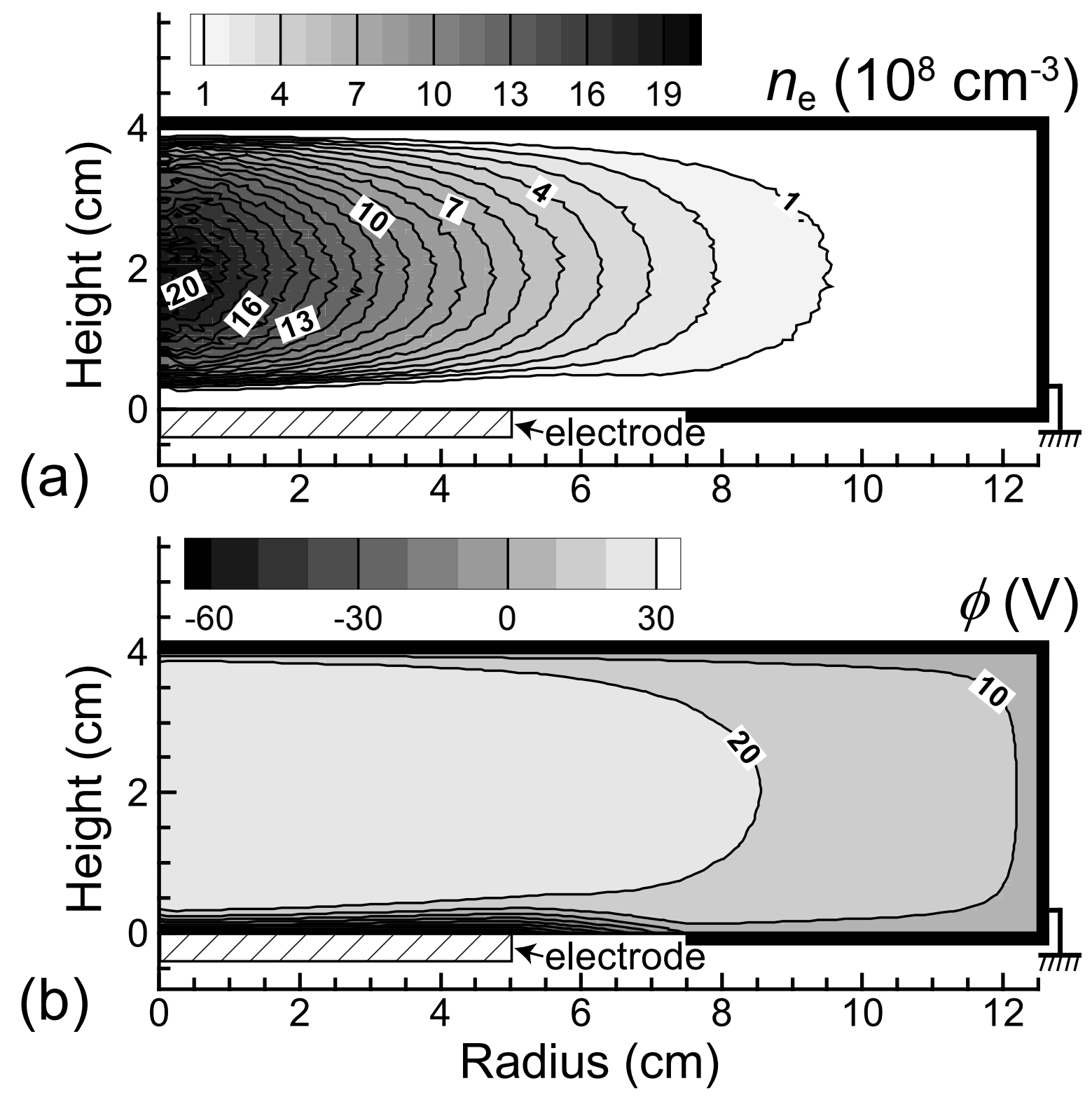

Figure 5 (width $7.7 \mathrm{~cm}$, scale 2/1)

Y. Takao et al. 

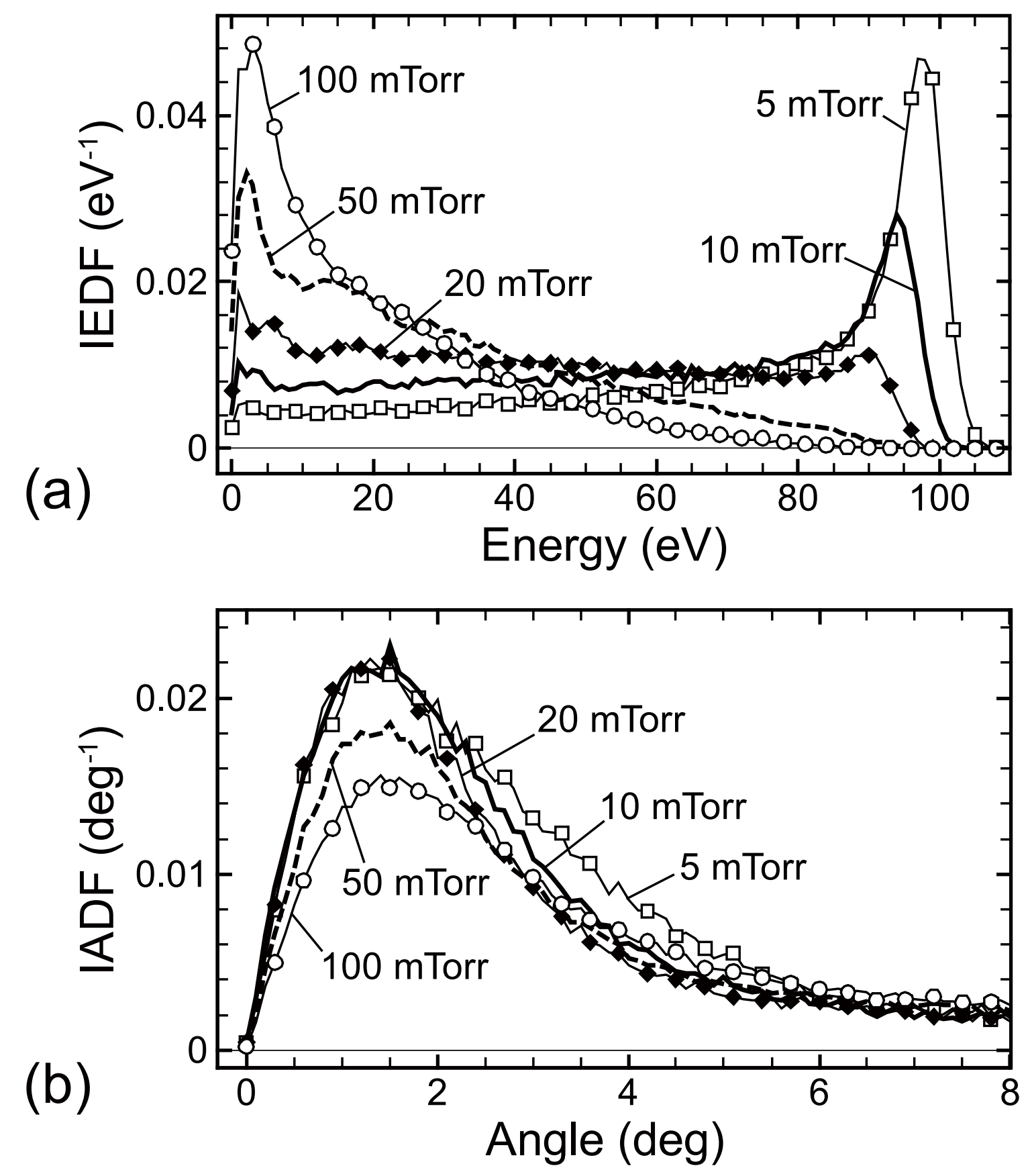

Figure 6 (width $7.7 \mathrm{~cm}$, scale 2/1)

Y. Takao et al. 


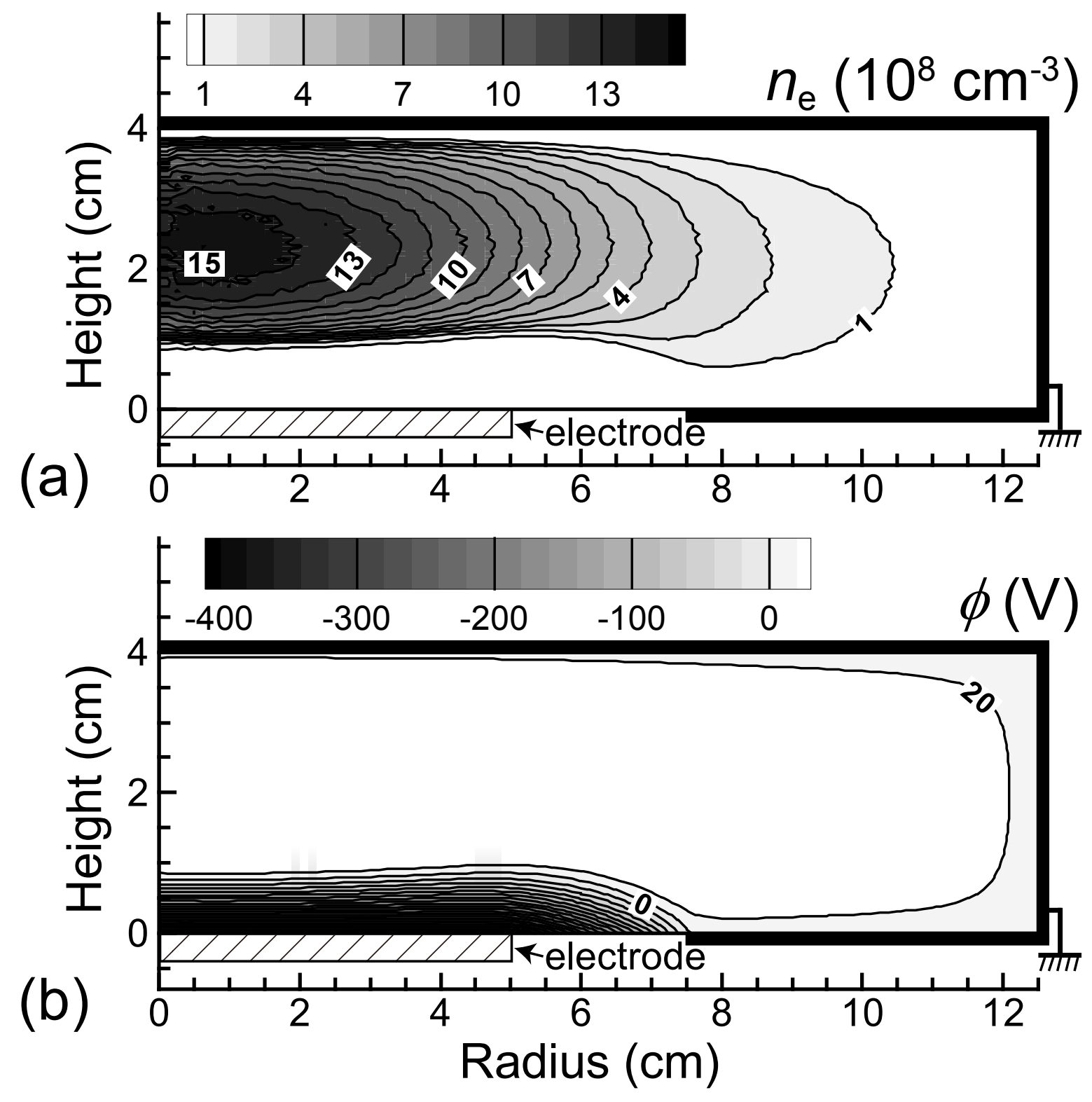

Figure 7 (width $7.7 \mathrm{~cm}$, scale 2/1)

Y. Takao et al. 

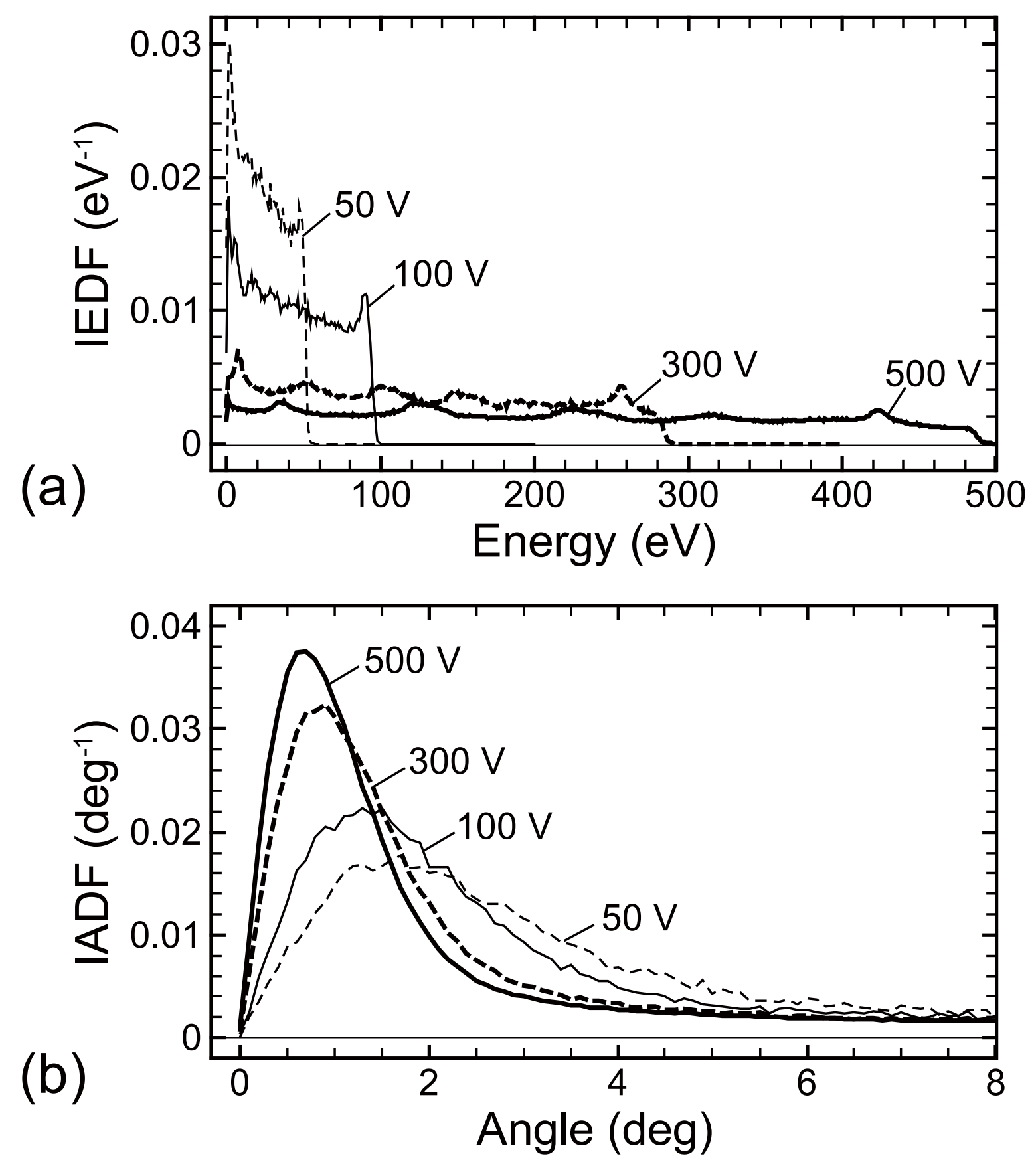

Figure 8 (width $7.7 \mathrm{~cm}$, scale 2/1)

Y. Takao et al. 


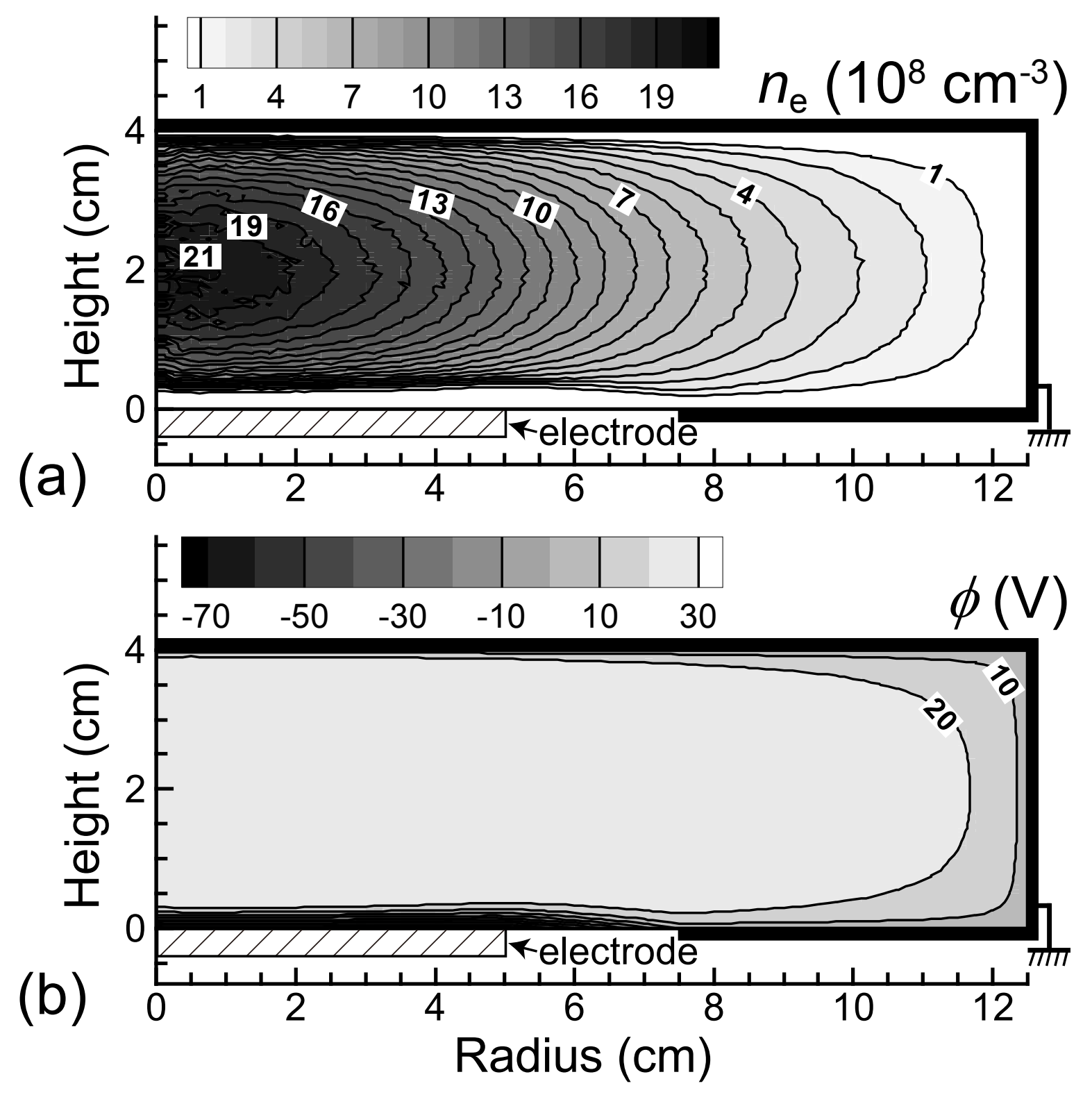

Figure 9 (width $7.7 \mathrm{~cm}$, scale 2/1)

Y. Takao et al. 

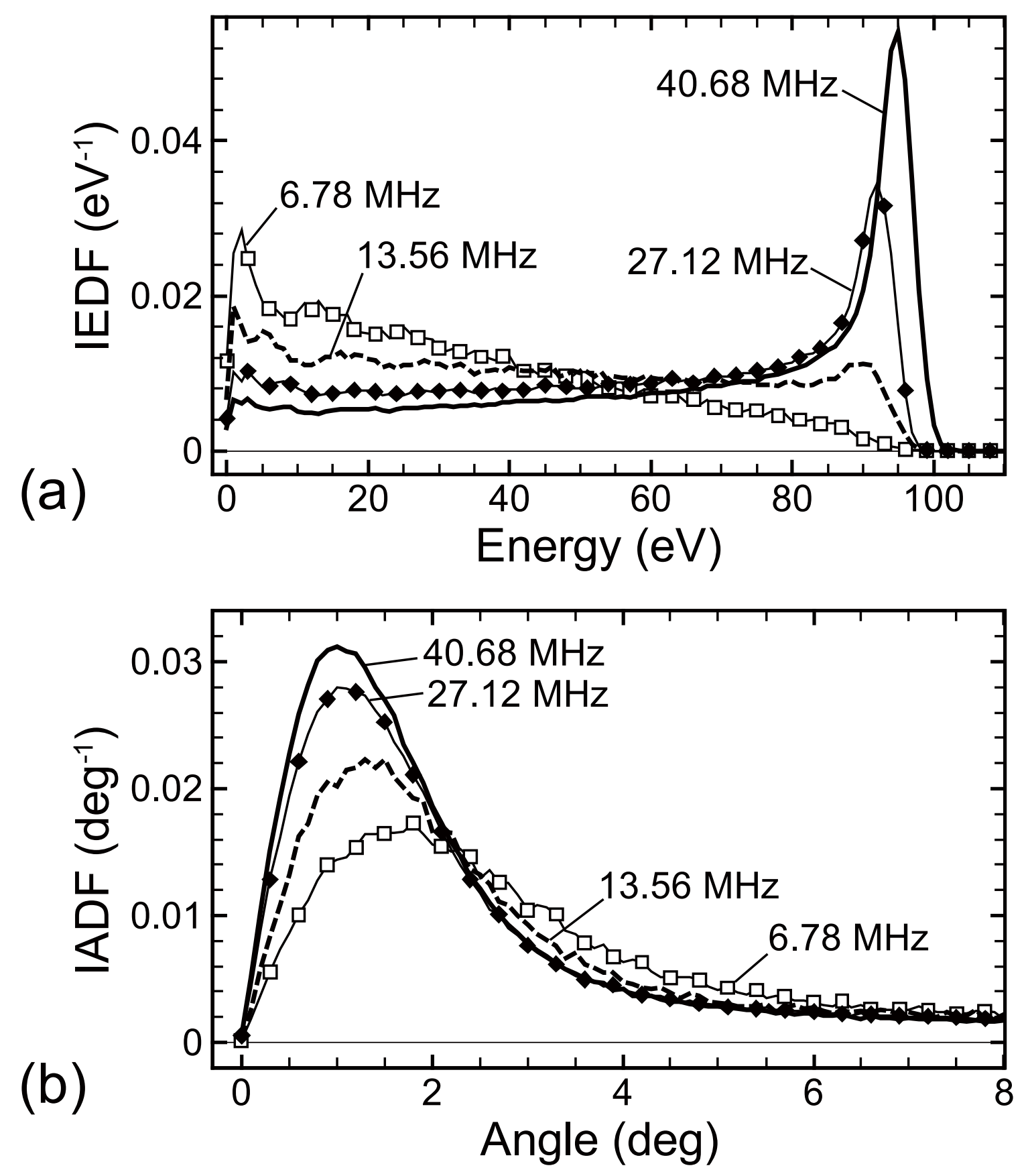

Figure 10 (width $7.7 \mathrm{~cm}$, scale 2/1)

Y. Takao et al. 

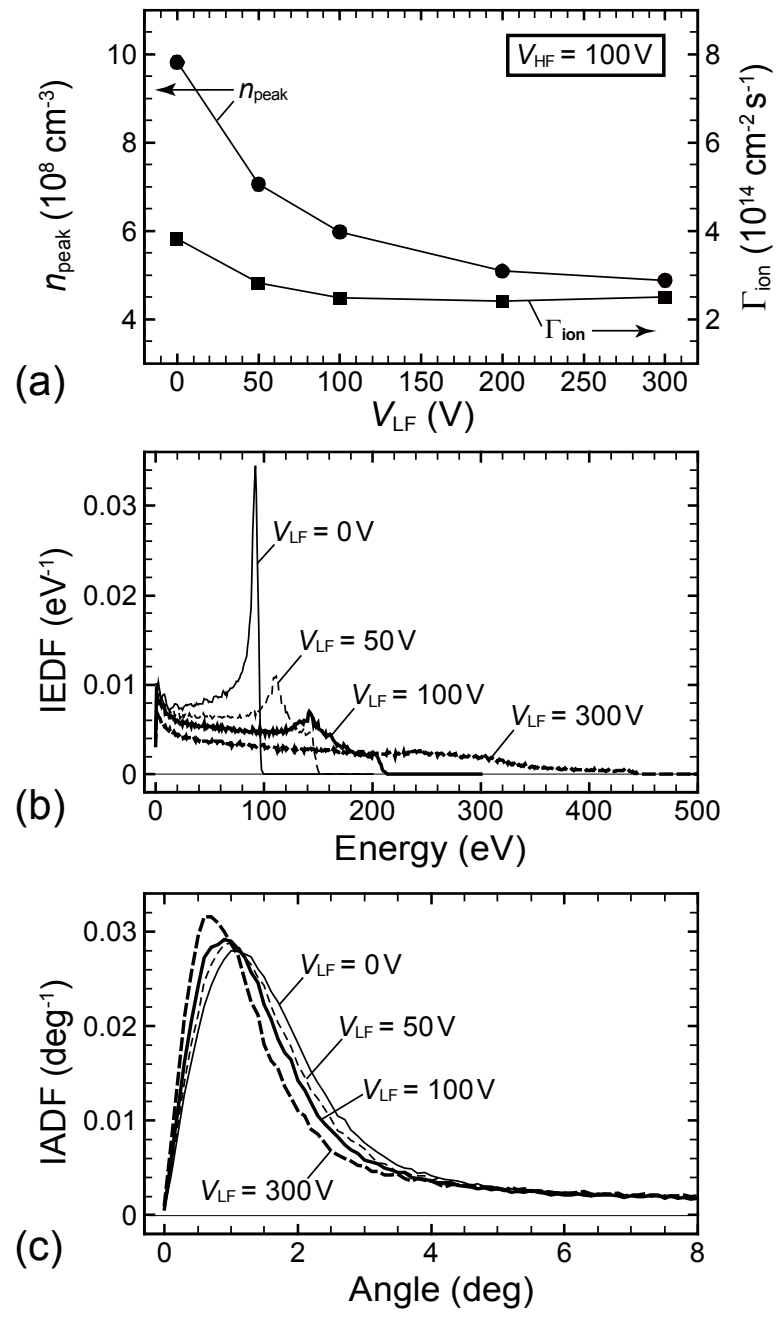

Figure 11 (width $7.8 \mathrm{~cm}$, scale 1/1)

Y. Takao et al. 